\title{
Identification and Characterization of the Myxococcus xanthus bsgA Gene Product
}

\author{
RONALD E. GILL* AND M. CRAMER BORNEMANN \\ Department of Microbiology and Immunology, University of Colorado Health Sciences Center, \\ Denver, Colorado 80262
}

Received 4 May 1988/Accepted 10 August 1988

\begin{abstract}
The bsgA mutants of Myxococcus xanthus are blocked at a very early stage of the developmental program. They fail to produce fruiting bodies or to sporulate under normal conditions but can be rescued by extracellular complementation in mixtures with wild-type cells. A bsgA-lacZ gene fusion was constructed and expressed in Escherichia coli. The resulting fusion protein, which has $\beta$-galactosidase enzyme activity, was partially purified by affinity chromatography and preparative polyacrylamide gel electrophoresis. The protein was used to immunize mice, which produced a hybridoma secreting monoclonal antibody that was specific for the bsgA gene product. The monoclonal antibody was used in Western blot (immunoblot) experiments to determine the apparent cellular location of the bsgA protein in $M$. xanthus and to compare the level of this protein at various times in the Myxococcus life cycle.
\end{abstract}

Myxococcus xanthus is a gram-negative soil bacterium which undergoes a complex response to starvation $(25,28)$. Upon encountering conditions of limiting nutrient, cells actively migrate to aggregation foci. As additional cells enter the aggregates, the structures enlarge to become fruiting bodies measuring a few tenths of a millimeter in diameter. Within the fruiting body, a portion of the cells differentiate into dormant, environmentally resistant myxospores. The myxospores may subsequently germinate to give rise once again to vegetative cells.

There is growing evidence to suggest that the control of the developmental process is, at least in part, dependent upon specific cell-cell interactions $(7,9,12,15,18,20)$. Four classes of conditional sporulation-deficient mutants have been isolated (9) which behave as if they are each defective in a different required intercellular signalling event and are given the phenotypic designations Asg, Bsg, Csg, and Dsg, (for A signal, etc.). These mutants are unable to sporulate when placed on appropriate starvation medium. However, they transiently regain the ability to sporulate if allowed to develop in a mixture with either wild-type cells or with cells of a different mutant class. There is no evidence that these results can be explained by genetic exchange between the cell types in the mixture or that there is any heritable change in the nonsporulating mutants. Instead, such behavior, termed extracellular complementation, is consistent with the hypothesis that the mutants are unable to produce the required developmental signal but remain able to respond to the signal when it is provided by another cell.

In the accompanying paper (8) we report the characterization of a group of Bsg mutants that belong to a single genetic complementation group, $b s g A$ (formerly $s s b A$ [7]). These nonsporulating mutants appear to arrest very early in development. We do not observe any indication of organized aggregation or fruiting body formation. To further characterize the $b s g A$ mutants, developmentally induced lac fusions were isolated and the effect of $b s g A$ mutations on their expression was determined. For each of the several developmental fusions which have now been tested $(7,15)$, the bs $g A$ mutants are deficient or show reduced levels of expres-

\footnotetext{
* Corresponding author.
}

sion. Under the conditions of our assay (7), extracellular complementation of the $b s g A$ mutants by wild-type cells will rescue both the sporulation defect and the ability of the $b s g A$ mutant cells to express at least certain developmentally induced fusions.

In addition to a developmental phenotype, we have reported an effect of mutations in the $b s g A$ gene on the behavior of vegetatively growing cells (8). Both wild-type and mutant cells form flat, swarming colonies on solid medium. However, when a swarming mutant and wild-type colony converge, the leading edge of the mutant colony appears to stop advancing and an abrupt ridge of cells forms at the edge of the colony. This behavior is the specific consequence of the interaction of a mutant and wild-type colony; it is not observed when either two wild-type or two mutant colonies converge.

As a step toward understanding the role of the $b s g A$ gene in the regulation of developmental gene expression, we report here the identification of the $b s g A$ gene product, its localization within $M$. xanthus cells, and the pattern of its expression during the vegetative and early developmental stages of the Myxococcus life cycle.

\section{MATERIALS AND METHODS}

Bacterial strains and media. Escherichia coli D1210 (26), an $\mathrm{HB} 101$ derivative that is $l a c I^{+} Z^{+} Y^{+}$recA56 hsdR hsdM rpsL leu thi pro, was used for routine transformations of ligation reactions for plasmid constructions and in the maxicell experiments described below. Plasmids were transformed into $E$. coli D1246 for Western blot (immunoblot) analysis of plasmid-encoded proteins. $E$. coli D1246 is D1245 $\left(\mathrm{F}^{\prime}\right.$ lacI $^{q} \mathrm{Z}$ proA $A^{+}$proB $\left.{ }^{+}\right)$; the lac $Z$ mutation on the $\mathrm{F}^{\prime}$ is a strong polar mutation and does not result in synthesis of a peptide that cross-reacts with the anti- $\beta$-galactosidase monoclonal antibody used in our Western blots. D1245 is an HB101 derivative that is $\Delta($ lacX74) rpsL recA56 hsdR hsdM leu thi. D1210 and D1246 were obtained from Joan Betz. $E$. coli cells were routinely grown in LB medium (4) supplemented, when appropriate, with ampicillin $(150 \mu \mathrm{g} / \mathrm{ml})$ and kanamycin $(40 \mu \mathrm{g} / \mathrm{ml})$. To derepress transcription from promoters regulated by lac repressor, isopropyl- $\beta$-D-thiogalactopyranoside (IPTG) (1 mM final concentration) was added 
to mid-log-phase cultures for $2 \mathrm{~h}$ before harvesting cells for $\beta$-galactosidase assays or Western blot analysis.

$M$. xanthus $\mathrm{M} 102$ is our single-colony isolate of fully motile, wild-type strain DK1622 obtained from D. Kaiser and D. Morandi. M252 is an bsgA301 mutant of M102 (7). It contains a 4-base-pair (bp) deletion at an SstII site within the bsgA gene. This mutation was constructed in vitro and transferred to the $M$. xanthus chromosome by gene replacement (8). The $M$. xanthus strains were routinely grown vegetatively in CTT medium (11) supplemented with kanamycin $(50 \mu \mathrm{g} / \mathrm{ml})$ when appropriate. Development was induced by using the submerged culture technique of Kuner and Kaiser (17), as modified by Kuspa et al. (18). In this technique, broth cultures of $M$. xanthus are dispensed into plastic petri dishes and incubated without agitation, during which time the cells adhere firmly to the bottom of the dish. Starvation conditions for the induction of the developmental program are initiated by pouring off the CTT medium, gently washing the adherent cells with sterile water, replacing the medium with MC7 (10 mM Tris [pH 7.0], $1 \mathrm{mM} \mathrm{CaCl}$ ), and incubating the plates at $30^{\circ} \mathrm{C}$. Under these conditions, fruiting body formation begins at approximately $12 \mathrm{~h}$ after induction.

Plasmid constructions. The general methods used for the formation, isolation, and characterization of recombinant plasmid DNA are those described by Maniatis et al. (21). All enzymes used in plasmid constructions were used according to the manufacturer's specifications. Restriction enzymes, linkers, and T4 ligase were from Boehringer Mannheim Biochemicals; mung bean nuclease and T4 polymerase were from Pharmacia Fine Chemicals. Restriction enzyme fragments used in ligations were routinely purified by agarose gel electrophoresis, electroelution of the desired fragment into dialysis tubing, and purification by NACS chromatography (Nucleic Acid Chromatography System; Bethesda Research Laboratories, Inc.) according to the manufacturer's specifications.

pREG1175, pREG1311, and pREG1312. pREG1175 is a pBR322 derivative that can be used for the formation of operon fusions to lacZ (Fig. 1). To construct pREG1175, a promoterless trp-lac fragment was inserted into pREG428 (Fig. 1). pREG428 is identical to pREG429 (whose properties are fully described in the accompanying paper [8]), except for the size of the fragment that encodes resistance to kanamycin. It is a pBR322-derived plasmid which contains the $A p^{r}$ determinant and the origin of replication (ori) from pBR322, the $\mathrm{Km}^{\mathrm{r}}$ determinant from $\mathrm{Tn} 5$, and the plasmid incompatibility region of bacteriophage $\mathrm{P} 1$, which allows efficient transduction of the plasmid by $\mathrm{P} 1$ from $E$. coli into Myxococcus cells. The $\mathrm{Km}^{\mathrm{r}}$ determinant was derived from Tn5 as a 1.5-kb HindIII-SalI fragment in which the HindIII site was converted to a BamHI site by the addition of a BamHI linker. The $\mathrm{Km}^{\mathrm{r}}$ fragment was inserted into BamHISall-cut $\mathrm{pBR} 322$. The $\mathrm{P} 1$ inc region was inserted as a 6.6-kilobase EcoRI fragment from bacteriophage P1, followed by removal of one of the EcoRI sites with T4 DNA polymerase as described below. To construct pREG1175, pREG428 was cut at its unique BamHI site, and the BamHI$B$ III promoterless trp-lac fragment from pMC903 (3) was inserted. The orientation of the lac fragment was determined by double digestions with EcoRI and BamHI. pREG1175 has lac oriented in a clockwise direction and was used for construction of operon fusions to lac; pREG1174 has lac oriented in a counterclockwise direction and was used in the construction of pREG1363. The trp-lac fragment contains an intact lacZ gene (including its own ATG and ribosome-
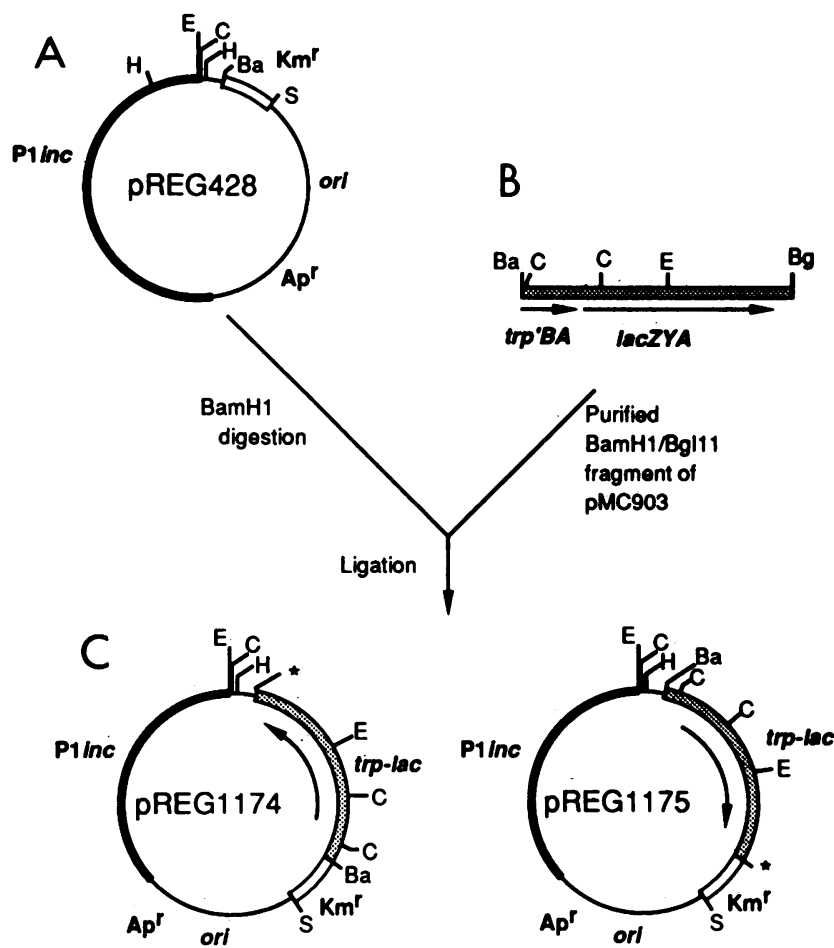

FIG. 1. Origin of plasmids pREG1174 and pREG1175. (A) Circular map of pREG428, showing the location of relevant restriction sites and structural features. Symbols: - , sequences derived from pBR322; $\square$, sequences derived from $\mathrm{Tn} 5$ and encoding resistance to kanamycin; $\mathbf{\square}$, DNA derived from bacteriophage $\mathrm{P} 1$, which encodes the $\mathrm{P} 1$ incompatibility region $(\mathrm{P} 1$ inc). (B) Linear representation of the BamHI-BglII promoterless trp-lac fragment derived from pMC903 (described in Materials and Methods). The location and orientation of the trp and lac genes contained on the fragment are indicated. Plasmid pREG429 was digested with BamHI and ligated to the purified trp-lac fragment to form pREG1174 and pREG175. Circular maps of pREG1174 and pREG1175 are shown in panel C. Symbols: tritis , trp fragment (the orientation of the trp and lac genes is indicated by the arrow); other symbols as in panel A. Restriction sites: Ba, BamHI; Bg, BglII; C, ClaI; E, EcoRI; *, hybrid BamHI-BglII site no longer recognized by either enzyme.

binding site) as well as $\operatorname{lac} Y$ and $\operatorname{lacA}$ but contains no promoter sequences capable of directing lac transcription (3). Therefore, expression of lac is dependent upon readthrough transcription from a promoter sequence cloned at the upstream $B a m H I$ site. The $\operatorname{trp}^{\prime} B A$ sequences located on the fragment, $5^{\prime}$ of lac, serve to terminate incoming translation so as not to interfere with initiation at the proper translational initiation of lacZ.

The construction of pREG1311 and pREG1312 are shown in Fig. 2. pREG1255 (8) is pREG428 containing a 12-kilobase EcoRI fragment of $M$. xanthus DNA which contains the entire $b s g A$ locus. pREG1311 was formed by inserting the indicated $M$. xanthus-derived BamHI-BglII fragment (fragment $A$ in Fig. 2A) from pREG1255 into the unique BamHI site of pREG1175. This fragment contains the portion of the bsgA locus to the left of the $B g$ III site and is in the orientation shown in Fig. 2B. pREG1312 was formed by inserting the indicated $B g$ lII-EcoRI fragment (fragment B in Fig. 2A) from pREG1255 into pREG1175. This fragment contains the portion of the $b s g A$ locus to the right of the $B g$ III site. For this construction, pREG1175 was completely digested with Bam $\mathrm{HI}$ and partially digested with EcoRI so as 
A

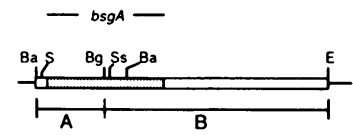

B
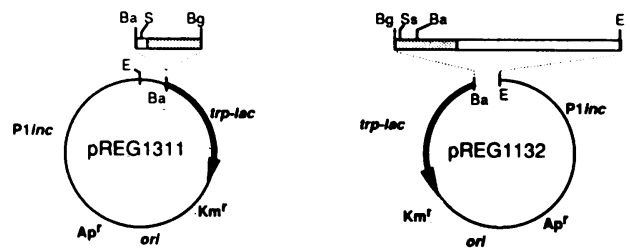

C

1) Integrated pREG1311

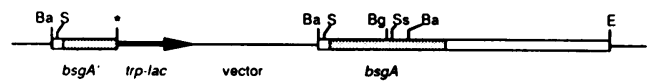

2) Integrated pREG1312

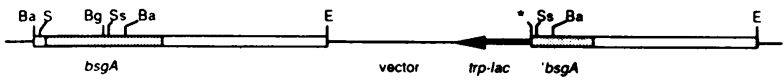

FIG. 2. Formation of bsgA-lacZ operon fusion in M. xanthus. (A) Representation of the $M$. xanthus bsgA locus ( $M$ itis) and its flanking DNA sequences, showing the location of relevant restriction sites. Fragments A and B were inserted into BamHI-digested and BamHI-EcoRI-digested pREG1175, respectively. The resulting plasmids, pREG1311 and pREG1312, respectively, are shown in panel $B$. The location and orientation of the insert fragments are shown relative to those of the trp and lac genes $(\rightarrow)$ of the pREG1174 parent plasmid. Transduction of pREG1311 and pREG1312 into $M$. xanthus results in the integration of the plasmid into the bacterial chromosome via homologous recombination between the cloned Myxococcus DNA and its chromosomal homolog. The structures of the integrated plasmids are shown in panel C. Restriction sites: Ss, SstII; S, SalI; others are as in Fig. 1.

to cut at only one of the two recognition sites for this enzyme in the plasmid. A fragment of appropriate size was eluted from an agarose gel, purified by NACS chromatography, and ligated to the similarly purified insert fragment.

Transfer of plasmid DNA from $E$. coli to $M$. xanthus by specialized P1 transduction is described in the accompanying paper (8). The specific activity of $\beta$-galactosidase from lac fusion-containing strains was determined as previously described (16).

pREG1340, pREG1344, and pREG1352. The plasmid pKK233-2 (1), described originally as an ATG-containing expression vector, was first modified to remove the BamHI, EcoRI, and SalI sites, which simplified our subsequent manipulations presented here and elsewhere. To remove these sites, the plasmid was cut with EcoRI and SalI, treated with T4 DNA polymerase to make flush ends, and circularized with T4 DNA ligase. A SalI site was then added downstream of the trc promoter by cutting with NcoI, treating with mung bean single-strand nuclease to remove the sticky ends, and ligating phosphorylated SalI linker to the blunt ends. This was followed by treatment with SalI to produce sticky ends, recircularization with T4 DNA ligase, and transformation into $E$. coli D1210 to form pREG1340 (Fig. 3). These manipulations had two important consequences: (i) a Sall site, which can be used for cloning, was introduced just downstream of the trc promoter; (ii) the ATG start codon located within the NcoI recognition sequence, which would otherwise be used as an efficient translational
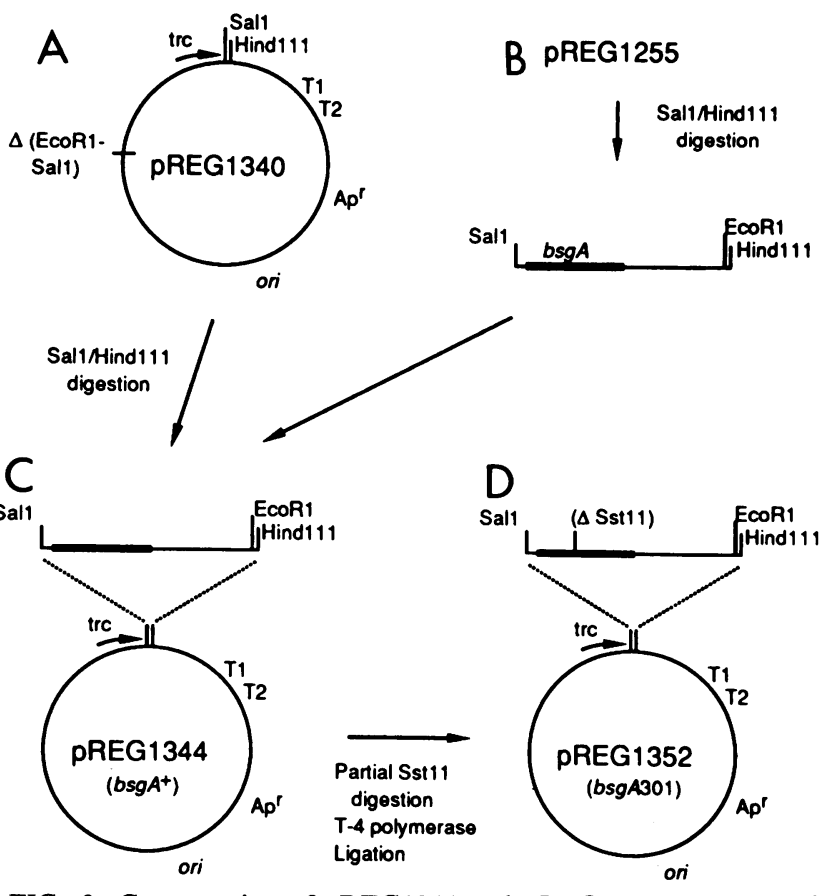

FIG. 3. Construction of pREG1344 and pREG1352. (A) Map of pREG1340, which was derived from pKK233 by the removal of nonessential DNA between its EcoRI and SalI sites and subsequent addition of a SalI linker downstream of the $t r c$ promoter (described in Materials and Methods). $\operatorname{trc}, \operatorname{trc}$ promoter; $\mathrm{T}_{1}$ and $\mathrm{T}_{2}$, transcriptional terminators. (B) SalI-HindIII fragment from pREG1255, which contains the entire bsgA locus ( $)$. The arrow indicates the presumed direction of transcription of the $b s g A$ gene. This fragment was inserted into SalI-HindIII-digested pREG1340 to form pREG1344 (C). The orientation of the bsgA fragment is shown relative to the direction of transcription from the trc promoter (indicated by the arrows). (D) Map of pREG1352, which is identical to pREG1344 except for the introduction of the bsgA301 mutation, a 4-bp deletion at an SstII site within the bsgA gene (designated $\Delta$ SstII on the map). The deletion was constructed by partial SstII digestion of pREG1344 and treatment with T4 DNA polymerase to remove the 3 ' overhanging sticky ends and circularization with T4 DNA ligase.

start site, was removed. This made it less likely that there would be interference with translational initiation at the normal start codon of the cloned genes.

pREG1344 was formed by inserting a SalI-HindIII fragment of pREG1255 into pREG1340 (Fig. 3). This fragment contains all of the $b s g A$ locus and is oriented such that the trc promoter on pREG1340 is just upstream of the $b s g A$ gene. This plasmid was stably maintained in $E$. coli D1210 $\left(\operatorname{lacl} I^{\mathrm{q}}\right)$ in LB medium. Induction of transcription from the trc promoter by IPTG or lactose and attempts to introduce the plasmid into $\mathrm{C} 600$ (lacI $^{+}$) resulted in marked plasmid instability. pREG1352 (Fig. 3) is identical to pREG1344, except it contains a 4-bp deletion at an SstII site within the $b s g A$ locus. The mutation was formed by cutting with SstII, removing the 3' overhanging sticky ends with T4 DNA polymerase, and recircularizing with T4 DNA ligase.

pREG1361, pREG1363, pREG1364, and pREG1366. pREG1361 (Fig. 4) contains an operon fusion between a truncated $b s g A$ gene and a downstream intact but promoterless $l a c Z$ gene. The fusion is efficiently transcribed from the upstream trc promoter and regulated by the lac repressor. pREG1361 was constructed by first digesting pREG1344 with BamHI and HindIII. The single fragment containing 

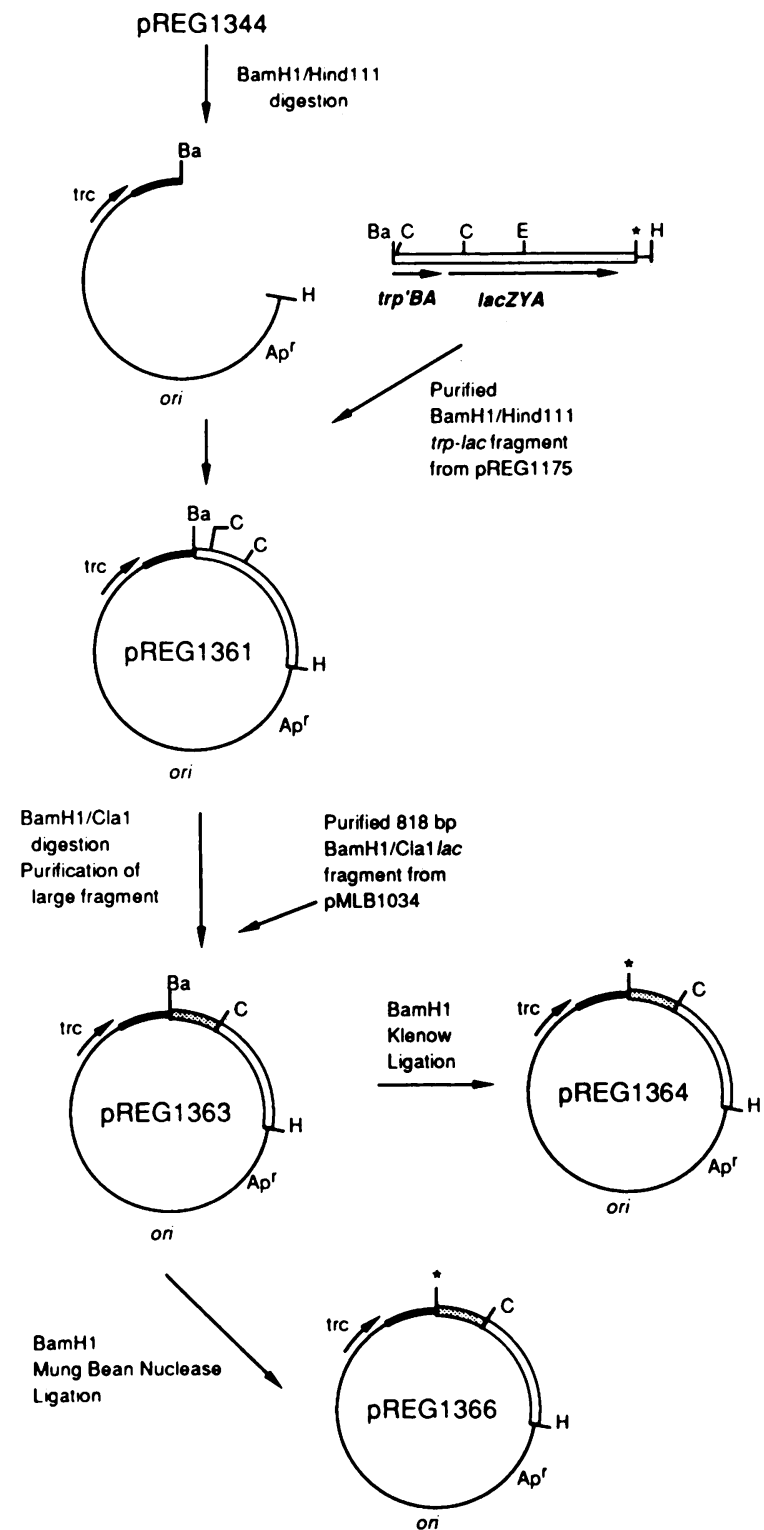

FIG. 4. Formation of a $b s g A-l a c Z$ gene fusion plasmid. The BamHI-HindIII trp-lac fragment of pREG1175 was inserted into pREG1344 to form pREG1361. Symbols: $\square$, trp-lac fragment; pREG340 sequences; - bsgA sequences. $\operatorname{trc}, \operatorname{trc}$ promoter from pKK233-2 (arrow designates direction of transcription from $\operatorname{trc}$ ). Restriction sites are designated as in Fig. 1. This plasmid contains a bsgA-lac operon fusion and results in the expression of wild-type $\beta$-galactosidase. pREG1361 was then digested with BamHI and ClaI, and the large fragment was purified. This removed the trp sequences and the first 279 amino acid codons of lacZ. In its place, an 818-bp BamHI-ClaI lac fragment from pMLB1034 ( was ) was inserted, in which the BamHI site is in a polylinker region adjacent to the ninth amino acid codon of $l a c Z$ and the ClaI site is at the analogous site at amino acid 279 of $l a c Z$. To generate plasmids in which the $b s g A-l a c Z$ junction was in each of the three possible reading frames, pREG1363 was cut with BamHI and treated with Klenow polymerase or mung bean nuclease and recircularized to generate pREG1364 (4-bp insertion) and pREG1366 (4-bp deletion), respectively. The asterisk designates the location of these BamHI site mutations.

the vector and the $5^{\prime}$ portion of the $b s g A$ locus was purified. This fragment was ligated to the BamHI-HindIII fragment containing the promoterless $\operatorname{trp}^{\prime} B A-l a c Z Y A$ genes from pREG1174. This particular lac fragment produces operon fusions to upstream promoters and results in the production of wild-type $\beta$-galactosidase.

The plasmid pREG1363 was formed by converting the operon fusion in pREG1361 to a $b s g A$-lacZ gene fusion, in which approximately $1,500 \mathrm{bp}$ of the $b s g A$ coding sequence is joined in the same translational reading frame to the ninth amino acid codon of lacZ. To construct pREG1363 (Fig. 4), the plasmid pREG1361 was first cut with BamHI and ClaI. BamHI cuts pREG1361 at the junction between the bsgA sequences and the trp-lac fragment. ClaI cleaves pREG1361 at two positions within the trp-lac fragment, once within $\operatorname{trp} A$ and once within the $\operatorname{lac} Z$ coding sequence. (A third recognition site is located in lac $Y$ but is not cleaved in dam methylated $E$. coli strains.) To create the desired gene fusion between bsgA and lacZ, an 818-bp BamHI-ClaI lacZ fragment from pMLB1034 (2) was inserted into the large BamHIClaI fragment of pREG1361. The BamHI site in the pMLB1034-derived fragment is located in a polylinker region immediately adjacent to the ninth amino acid codon of laçZ; the $C l a \mathrm{I}$ site is at the same site in $l a c Z$ coding sequence as in pREG1361. This manipulation removes from pREG1361 the translational stops preceding $l a c Z$ and the ribosome-binding site and translational start of $l a c Z$ itself. In its place is the internal lac $Z$ fragment from pMLB1034, so that the upstream $b s g A$ coding sequence is now situated adjacent to the lacZ ninth amino acid codon, and the remainder of the lac $Z$ coding sequence is restored. Since successful formation of a fusion protein depends on having the $b s g A$ and $l a c Z$ coding sequences in the same translational reading frame, each of the two additional reading frames at the $b s g A-l a c Z$ junction was also constructed (Fig. 4). pREG1363 (the first reading frame) was cut with BamHI and treated with mung bean single-stranded nuclease (pREG1366) or with Klenow enzyme (pREG1364) to introduce a 4-bp deletion or insertion, respectively, at the junction between the $b s g A$ and $l a c Z$ coding regions. Only pREG 1363 produced detectable $\beta$ galactosidase when induced with IPTG and so likely contains the fusion in the correct reading frame.

Maxicells. $E$. coli maxicells were used to identify plasmidencoded polypeptides by a modification of the standard protocol (27). The E. coli host was strain D1210, a lacI ${ }^{\mathrm{q}}$ lac $Z Y A^{+}$derivative of HB101. Cells were grown to exponential phase (optical density at $550 \mathrm{~nm}, 0.2$ ) in Davis minimal medium (Difco Laboratories) supplemented with $0.2 \%$ Casamino Acids (Difco), thiamine $(50 \mu \mathrm{g} / \mathrm{ml}), 0.2 \%$ glucose, and ampicillin $(100 \mu \mathrm{g} / \mathrm{ml})$. Cells were UV irradiated for $2 \mathrm{~min}$, a time which was empirically found to give satisfactory detection of plasmid-encoded peptides with minimal interference by chromosomally encoded peptides. After irradiation, cells were shaken at $37^{\circ} \mathrm{C}$ for $90 \mathrm{~min}$ in the dark, at which time cycloserine was added to $200 \mu \mathrm{g} / \mathrm{ml}$. After 14 $\mathrm{h}$ of additional shaking, fresh cycloserine was added, and the culture was shaken for an additional $2 \mathrm{~h}$. Cells were then harvested, washed in unsupplemented Davis medium, and suspended in Davis medium containing thiamine, glucose, and the required amino acids proline and leucine (each at 20 $\mu \mathrm{g} / \mathrm{ml})$. After the culture was shaken at $37^{\circ} \mathrm{C}$ for $45 \mathrm{~min}$, IPTG ( $1 \mathrm{mM}$ final concentration) and ${ }^{14} \mathrm{C}$-amino acids (specific activity, $1.79 \mathrm{mCi} / \mathrm{mg} ; 10 \mu \mathrm{Ci} / \mathrm{ml}$, final concentration; ICN Pharmaceuticals) were added. Cells were incubated an additional $2 \mathrm{~h}$; washed in unsupplemented Davis medium, and boiled in sodium dodecyl sulfate-polyacrylamide gel 
electrophoresis (SDS-PAGE) sample buffer (19). Samples were stored at $-70^{\circ} \mathrm{C}$ until used.

Protein analysis. Total cell protein was fractionated by SDS-PAGE (19). Cells were lysed by boiling for $3 \mathrm{~min}$ in sample buffer. The methods used in Western blot analysis for transferring protein bands to nitrocellulose (7), blocking the filters (13), and reacting with the primary antibody (7) have been described. The relative quantity of $b s g A$ protein in different lanes of the same Western blot filter was estimated by densitometry. The monoclonal anti $\beta$-galactosidase antibody was from Boehringer. Bands that reacted with the primary antibody were visualized by reacting the filters with alkaline phosphatase-conjugated goat anti-mouse immunoglobulin G (Promega Biotec, Inc.) and developing with the color-developing substrates nitroblue tetrazolium and 5-bromo-4-chloro-3-indolyl phosphate as specified by the supplier (Promega). For detection of total protein transferred to nitrocellulose, filters were stained with India ink (10). ${ }^{14} \mathrm{C}$-labeled peptides were detected after SDS-PAGE by fluorography with $\mathrm{En}^{3} \mathrm{Hance}$ as specified by the supplier (New England Nuclear Corp.).

Isolation of monoclonal-antibody-producing hybridomas. The bsgA-lacZ fusion protein was produced in $E$. coli D1246(pREG1363) after induction with IPTG (1 mM). Cells were disrupted by sonication, and the enzymatically active fusion protein was partially purified by affinity chromatography on $p$-aminophenyl- $\beta$-D-thio-galactopyranoside-agarose (Sigma Chemical Co.) by the method of Germino et al. (6), followed by preparative SDS-PAGE. The band of interest was cut out of the Coomassie blue-stained gel, macerated by extrusion through a 26-gauge needle in a minimum volume of sterile saline, and injected subcutaneously with Freund complete adjuvant (for the first inoculation) or incomplete adjuvant (subsequent inoculations) into BALB/c mice. Preparation of spleen cells, cell fusions, and isolation of hybridoma cell lines have been described (14). Culture supernatants from individual hybridomas were assayed in duplicate by an enzyme-linked immunosorbent assay for the presence of antibody reacting with $\beta$-galactosidase or with the purified bsgA-lac $Z$ fusion protein. Hybridomas that reacted with the fusion protein but not with authentic $\beta$-galactosidase were presumed to produce a monoclonal antibody specific for the Myxococcus portion of the bsgA-lacZ fusion protein. These hybridomas were cloned, and culture supernatants from one such cell line was used as a source of anti-bsgA antibody for Western blotting experiments. The proper specificity of this antibody was assured by its reactivity in Western blots with $E$. coli carrying the cloned $b s g A$ gene and the bsgA-lacZ fusion but not with plasmidless $E$. coli and by reactivity with wild-type $M$. xanthus but not with $M$. xanthus M252, which has the bsgA301 frameshift mutation (see Results).

$M$. xanthus cell fractionations. The periplasmic fraction was released from intact cells by osmotic shock as previously described for $M$. xanthus (5). For the preparation of a membrane fraction, intact vegetative cells were lysed by sonication or by three cycles of freeze-thaw lysis, followed by centrifugation at $100,000 \times g$ for $1 \mathrm{~h}$ in a Beckman Ti70.1 rotor. The resulting pellet constitutes the crude membrane fraction; the supernatant is the soluble fraction.

The $b s g A$ protein was extremely sensitive to protease degradation in these extracts. Inclusion of $5 \mathrm{mM}$ EDTA in the lysis buffer ( $30 \mathrm{mM}$ Tris [pH 7.5], $5 \mathrm{mM}$ EDTA) virtually eliminated this problem, and this buffer was used throughout the $M$. xanthus experiments described here. However, because this level of EDTA also results in sufficient damage of the cell wall to lyse intact cells (unpublished observations), there was concern that certain proteins may artifactually dissociate from membranes under these conditions. Therefore, phosphoramidon (Boehringer), a nonchelating metalloprotease inhibitor, was used in parallel experiments and gave a similar proportion of $b s g A$ protein fractionating with the membrane and soluble fractions (data not shown).

The crude membrane fraction was further fractionated by equilibrium sucrose gradient centrifugation as previously described $(23,24)$. Two major bands and one minor band of intermediate density were observed. The bands were collected and found to correspond to the density of separated $M$. xanthus inner and outer membranes (the major bands, at densities of 1.22 and $1.16 \mathrm{~g} / \mathrm{ml}$, respectively) and hybrid membranes (intermediate band, at a density of $1.18 \mathrm{~g} / \mathrm{ml}$ ) previously reported by Orndorff and Dworkin (23).

\section{RESULTS}

Construction of a bsgA-lacZ operon fusion. We report in the accompanying paper the cloning and genetic characterization of the $M$. xanthus gene that is now designated $b s g A(8)$. However, our preliminary attempts to use $E$. coli maxicells to identify the product of the $b s g A$ locus when cloned into pBR322 were unsuccessful (data not shown). To investigate the possibility of increasing the level of bsgA expression in $E$. coli, we first determined the direction of its transcription by using $l a c Z$ operon fusions.

A partial restriction map of the $b s g A$ locus and its flanking DNA is shown in Fig. 2A. The location of the bsgA gene was determined by analysis of in vitro-constructed mutations, including a four-base insertion at the unique $B g$ III site (8). The two plasmids shown in Fig. 2B were constructed to determine the direction of transcription through this BglII site. In these plasmids, DNA to the left of the BglII site, (pREG1311) or to the right of the BgIII site, (pREG1312) was cloned into pREG1174 (Fig. 2) (see Materials and Methods) upstream of a promoterless lac $Z$ reporter gene so as to form a bsgA-lacZ operon fusion. Since these plasmids are incapable of replicating in $M$. xanthus, their introduction into these cells will result in the integration of the plasmid into the bacterial chromosome by recombination between the cloned DNA and its chromosomal homologue. The resulting sequence arrangements are shown in Fig. 2C. For each of the two integrated plasmids, the promoterless lac $Z$ sequences would be orientated such that its expression was dependent upon transcription from a Myxococcus promoter in the adjacent DNA and could not be transcribed by readthrough from plasmid promoters. Therefore, expression of lac $Z$ in these cells is expected to be a reliable indicator of the presence of a promoter, presumably the bsgA promoter, in the adjacent Myxococcus sequences. If transcription proceeded through the $b s g A$ locus from left to right (given the orientation in Fig. 2A), then Myxococcus strains having an integrated pREG1311 plasmid should express $\beta$-galactosidase. However, if transcription proceeded right to left, then only strains containing the integrated pREG1312 plasmid would be expected to express $\beta$-galactosidase.

The plasmids pREG1311 and pREG1312 were each transferred to wild-type $M$. xanthus M102 by P1 transduction. The specific activities of the $\beta$-galactosidases from exponentially growing cells of the resulting strains were determined. Only the integrated pREG1311 plasmid resulted in expression of significant levels of $\beta$-galactosidase $(365 \mathrm{nmol}$ of $o$-nitrophenol per min per mg protein, compared with 12 $\mathrm{nmol}$ of $o$-nitrophenol per min per $\mathrm{mg}$ of protein for pREG1312). These data indicate that transcription proceeds from left to right in Fig. 2A. 


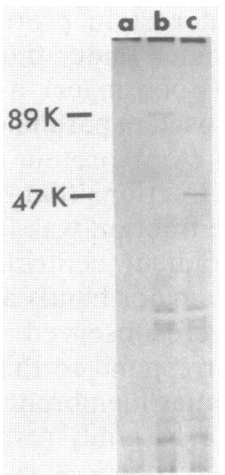

FIG. 5. Identification of the $b s g A$ protein product labeled in $E$. coli maxicells. $E$. coli maxicells were prepared, labeled with ${ }^{14} \mathrm{C}$ amino acids, and solubilized as described in Materials and Methods. Proteins were separated on an 8\% SDS-polyacrylamide gel; labeled bands were detected by fluorography. Lanes a, ( $E$. coli strains): D1210(pREG1340); b, D1210(pREG1344); c, D1210(pREG1352). The positions of the 89,000 -dalton $b s g A$ protein and truncated 47,000 -dalton $b s g A 301$ peptide are indicated.

Detection of the protein encoded by cloned bsgA locus in $E$. coli maxicells. When the SalI-EcoRI fragment containing the bsgA locus (Fig. 2A) was cloned into pBR322, no proteins that could be attributed to the $b s g A$ locus were detected in $E$. coli maxicells (data not shown). To enhance transcription of the locus, the SalI-EcoRI fragment was cloned into pREG1340, a derivative of the expression vector pKK233-2 (Fig. 3) (see Materials and Methods). The pREG1340 vector contains the trc promoter, a strong $E$. coli promoter from which transcription can be regulated by the lac repressor. In the resulting recombinant plasmid, pREG1344, the $b s g A$ locus was positioned in the correct orientation so that its transcription could be directed by the trc promoter (Fig. 3).

Proteins synthesized by IPTG-induced $E$. coli maxicells carrying pREG1344 are shown in Fig. 5. A prominent 89,000-dalton peptide was labeled in these cells but not in cells carrying only the vector (compare lanes $a$ and $b$ in Fig. 5). The bsgA locus, which is estimated to be about 2.4 kilobases (8), would be expected to encode a single protein of approximately this size.

To determine whether the 89,000 -dalton peptide was indeed the product of the $b s g A$ locus, we examined the proteins produced by cells carrying the $b s g A$ mutant plasmid pREG1352. This plasmid was formed by introduction of a 4-bp deletion at the SstII site within the $b s g A$ locus of pREG1344 (Fig. 3). This mutation, which has previously been shown in $M$. xanthus to result in a BsgA mutant phenotype $(7,8)$, would be expected to cause a shift in the bsgA translational reading frame and therefore result in the synthesis of a protein of altered size. Proteins labeled in IPTG-induced maxicells containing the pREG1352 mutant plasmid are shown in Fig. 5 (lane c). The 89,000-dalton peptide is absent from this track. Instead, there is a new 47,000-dalton peptide, presumably the truncated form of the wild-type $b s g A$ protein.

Identification of a bsgA-protein-reactive monoclonal antibody. We next wished to generate an antibody reagent suitable for detecting the bsgA protein in Myxococcus cell extracts. To facilitate purification of material suitable for immunization, we constructed the plasmid pREG1363, which encodes a $b s g A$-lacZ fusion protein. The origin of pREG1363 is shown in Fig. 4 and described in Materials and Methods. The bsgA-lacZ gene fusion was transcribed by the

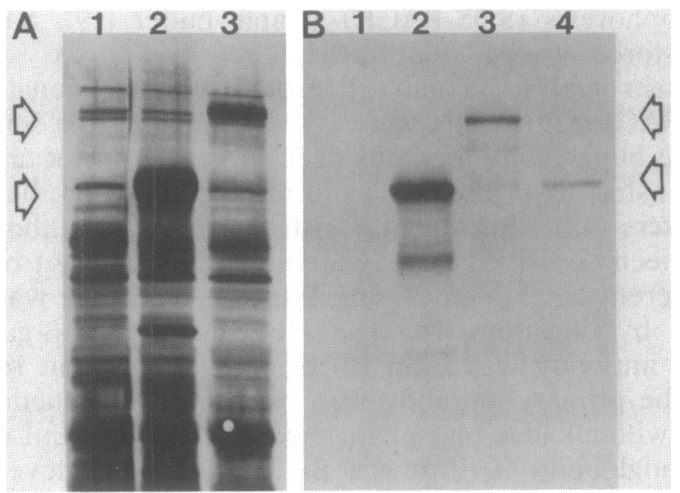

FIG. 6. Demonstration of a $b s g A-l a c Z$ protein fusion expressed in $E$. coli. Whole cell extracts of plasmid-containing $E$. coli were separated by SDS-PAGE, and the protein was transferred to nitrocellulose (Materials and Methods). (A) India ink stained filter. Lanes (E. coli strains): 1, D1246; 2, D1246(pREG1361); 3, D1246 (pREG1363). The position of authentic $\beta$-galactosidase in lane 2 and the $b s g A-l a c Z$ fusion protein in lane 3 are indicated by the arrows. (B) Western blot reacted with anti- $\beta$-galactosidase monoclonal antibody. Lanes: 1 through 3 , as in panel A; 4, E. coli D1210 $\left(\operatorname{lac} Z^{+}\right)$.

trc promoter and regulated by the lac repressor. The $5^{\prime}$ end of the bsgA-lacZ gene was contained on a SalI-to-BamHI restriction fragment that encoded approximately 1.5 kilobases of the $5^{\prime}$ portion of the $b s g A$ coding sequence. This segment was linked at its $3^{\prime}$ end, in the correct translational reading frame, to the ninth amino acid codon of an otherwise intact lac $Z$ gene.

The data in Fig. 6A show the presence of a prominent, unique, high-molecular-weight protein in stained gels of IPTG-induced cells carrying pREG1363 (lane 3). The size of the protein (approximately 170,000 daltons) was consistent with the expected size of the $b s g A-l a c Z$ fusion protein. It was not seen in plasmidless cells (lane 1), or in cells carrying pREG1361 (lane 2), the precursor of pREG1363. Instead, plasmid pREG1361, which encodes a bsgA-lacZ operon fusion, resulted in the synthesis of a unique, prominent protein which migrated, as expected, with authentic $\beta$ galactosidase. Additional evidence to confirm the identity of the fusion protein is shown by the Western blot in Fig. 6B. The 170,000-dalton protein reacted with anti $\beta$-galactosidase antibody (Fig. 6B, lane 3 ) and was clearly larger than authentic $\beta$-galactosidase produced by $E$. coli carrying pREG1361 (lane 2) or by strain D1210 (lane 4).

Although it has not been shown by DNA sequencing that the $b s g A$ and $l a c Z$ coding sequences in pREG1363 are indeed in the same translational reading frame, the data presented in Fig. 6 are consistent with the synthesis of a bsgA-lac $Z$ protein fusion of the expected size. Furthermore, two derivatives of pREG1363 were constructed (Fig. 4) in which the reading frame at the $b s g A$-lac $Z$ junction was altered by the addition (pREG1364) or deletion (pREG1366) of $4 \mathrm{bp}$. Neither of these two alternative reading frames resulted in the synthesis of significant levels of $\beta$-galactosidase activity $(<0.5 \%$ of that synthesized by pREG1363) or resulted in the production of a protein with the expected size of the fusion protein that could be detected on Western blots with anti- $\beta$-galactosidase antibody (data not shown).

The $b s g A$-lac $Z$ fusion protein was partially purified from sonicates of $E$. coli D1246(pREG1363) by $p$-aminophenyl- $\beta$ D-thiogalactoside-agarose affinity chromatography (6), followed by preparative PAGE. The 170-kilodalton band was 


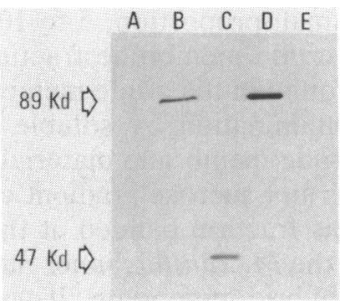

FIG. 7. Specificity of the $b s g A$-specific monoclonal antibody. Western blot of total cell extracts separated by SDS-PAGE and transferred to nitrocellulose membrane. The filter was reacted with tissue culture supernatant (diluted 1:200) from a $b s g A$-specific, monoclonal-antibody-producing hybridoma. Lanes: A, E. coli D1246; B, E. coli D1246(pREG1344); C, E. coli D1246(pREG1352); D, M. xanthus M102 (wild type); E, M. xanthus M252 (bsgA301).

excised from the Coomassie blue-stained preparative gel and used to immunize mice for production of hybridomas producing monoclonal antibody. Individual hybridomas were screened by an enzyme-linked immunosorbent assay for production of antibody specific for the Myxococcus portion of the 170-kilodalton fusion protein. Culture supernatants were tested in parallel for reactivity with the partially purified 170-kilodalton fusion protein and for reactivity with purified $\beta$-galactosidase. Culture supernatants from one of the hybridomas which reacted with the fusion protein but not authentic $\beta$-galactosidase was used for subsequent experiments.

The specificity of the monoclonal antibody for the product of the cloned $b s g A$ gene was verified by Western blot analysis (Fig. 7). The antibody detected an 89,000-dalton protein in IPTG-induced $E$. coli carrying pREG1344 (lane B), which encoded a wild-type $b s g A$ gene under transcriptional control of the trc promoter. This band was not seen in plasmidless cells (lane A) or in E. coli carrying the bsgA30I mutant plasmid pREG1352 (lane C). Instead, pREG1352 resulted in the appearance of a unique reactive band at 47,000 daltons, presumably a truncated form of the bsgA protein. These results are in complete agreement with the maxicell experiments reported above.

As a further test of its specificity, we have used the presumptive $b s g A$-specific monoclonal antibody in Western blots of $M$. xanthus total cell protein. This antibody did apparently detect authentic $b s g A$ protein in vegetatively growing Myxococcus cells (Fig. 7, lane D). The size of the protein appeared identical to that produced by $E$. coli expressing the cloned gene (compare lanes B and D in Fig. 7). Furthermore, this protein was not present in the bsgA301 mutant strain M252 (lane E).

Comparison of the level of $b s g A$ protein at various times during the $M$. xanthus life cycle. Mutations in the $b s g A$ gene are known to have phenotypic manifestations during vegetative growth of the cells (8) as well as during the developmental portion of the Myxococcus life cycle. Therefore, it is of some interest to determine when during its life cycle and in what relative quantity this protein is present. Figure 8 shows the results of a Western blot containing total cell extracts of mid-log-phase, vegetatively growing cells (lane A) and extracts of cells harvested at various times for $12 \mathrm{~h}$ after induction of development (lanes B through J). By comparing the relative intensity of the bands in Fig. 8, it can be seen that there was very little change in the level of $b s g A$ protein during this time interval. Densitometer measurements of relative band intensity indicated that there was a gradual twofold drop in the amount of protein detected

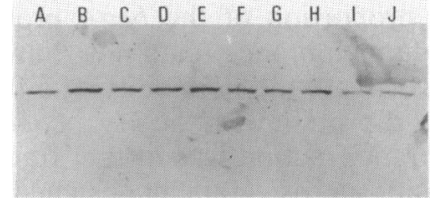

FIG. 8. Comparison of $b s g A$ protein levels in vegetative and early developmental cells. $M$. xanthus M102 was grown in submerged culture, and development was induced with MC7 buffer. Cells were harvested just before induction (vegetative cells, lane A), and at the following times after induction (lanes): B, $0.5 \mathrm{~h} ; \mathrm{C}, 1 \mathrm{~h} ; \mathrm{D}$, 1.5 h; E, 2 h; F, 4 h; G, 6 h; H, 8 h; I, 10 h; J, 12 h. For each time point, three plates were harvested, pooled, and boiled in SDSPAGE sample buffer. After SDS-PAGE, the separated proteins were transferred to nitrocellulose and reacted with the $b s g A$-specific monoclonal antibody.

during this 12-h interval (compare lanes $\mathrm{A}$ and $\mathrm{J}$ ). We certainly cannot rule out the possibility that this decrease is artifactual, due to increased proteolytic degradation in the cell extracts. We have found that the $b s g A$ protein is exceedingly susceptible to proteases in these extracts and that the general level of protease activity increases as the cells enter into development (unpublished observations).

Cellular localization of the bsgA protein. To determine the cellular localization of the $b s g A$ protein, exponentially growing Myxococcus cells were fractionated as described in Materials and Methods. Most, if not all, of the protein was found in the cytoplasmic fraction (Fig. 9). There was no detectable bsgA protein either in the culture supernatant, (lanes A and B) or released from the periplasmic space of osmotically shocked cells (lanes C and D). Furthermore, only 5 to $10 \%$ of the protein was found in the $100,000 \times g$ crude membrane pellet (lanes $\mathrm{E}$ and $\mathrm{F}$ ).

Since the proportion of $b s g A$ protein that was found in the membrane pellet was quite small, it is reasonable to simply suspect cross-contamination with the cytoplasmic fraction. However, it is also possible that the bsgA protein is only loosely associated with the membrane and becomes dissociated under the conditions used for preparation of the cell extract. This situation has been reported for the $\operatorname{secA}$ protein of $E$. coli (22). In that instance, the membrane-protein association of the $\sec A$ protein could be stabilized by the addition of $0.1 \mathrm{M} \mathrm{NaCl}$ to the lysate. Increasing the ionic strength of the Myxococcus lysates, however, had no effect

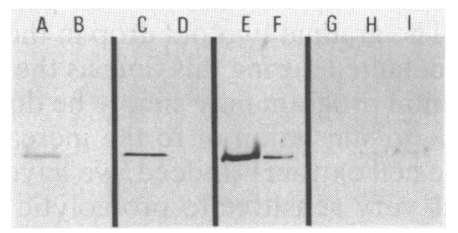

FIG. 9. Localization of the bsgA protein in Myxococcus cells. Cells were fractionated as described in Materials and Methods, and the $b s g A$ protein was detected by Western blot analysis by using the bsgA-specific monoclonal antibody. Lanes separated by white spaces represent separate experiments. An equal number of cell equivalents of protein was loaded onto lanes $A$ through $\mathrm{D}\left(5 \times 10^{7}\right.$ cells per lane) and $E$ through $I\left(2 \times 10^{8}\right.$ cells per lane $)$. Lanes: $A$, whole cells; B, cell-free culture supernatant; C, osmotically shocked whole cells; D, osmotic shock supernatant (periplasmic fraction); E, soluble fraction from $100,000 \times g$ centrifugation; $F$, crude membrane fraction from $100,000 \times g$ centrifugation; $G, 1.16-\mathrm{g} / \mathrm{ml} \mathrm{mem}-$ brane band (outer membrane fraction); $\mathrm{H}, 1.18-\mathrm{g} / \mathrm{ml} \mathrm{membrane}$ band (hybrid inner-outer membrane fraction); I, 1.22-g/ml membrane band (inner membrane fraction). 
on the relative proportion of the $b s g A$ protein that partitioned with crude membranes (data not shown).

To further investigate the possibility of a functional association of the $b s g A$ protein with the Myxococcus membrane, the crude membrane fraction was further fractionated on an equilibrium density sucrose gradient designed to separate $M$. xanthus inner, outer, and hybrid membranes. The three membrane bands were removed from the gradient and assayed for $b s g A$ protein by Western blot analysis. Interestingly, the portion of the $b s g A$ protein in the crude membrane fraction was associated with material banding at the density of inner membranes $(1.22 \mathrm{~g} / \mathrm{ml}$; Fig. 9, lane I) and hybrid membranes $(1.18 \mathrm{~g} / \mathrm{ml}$; lane $\mathrm{H})$. It was not detected in either the outer membrane band $(1.16 \mathrm{~g} / \mathrm{ml}$; lane $\mathrm{G})$ or at the position of $\beta$-galactosidase, a representative cytoplasmic protein (data not shown). Although these data do not prove a functional association of this portion of the $b s g A$ protein with the membrane, they leave open that possibility.

\section{DISCUSSION}

In this report, we described the construction and expression of a bsgA-lacZ gene fusion, the purification of the resulting fusion protein, and its use as an immunogen to raise antibody to the $b s g A$ protein. This strategy has been successful in raising a polyclonal rabbit serum (data not shown) as well as the mouse-derived monoclonal-antibody-producing hybridomas used in these experiments. It is of some interest to note the unexpected observation that, among the 12 animals that have been immunized (two rabbits and 10 BALB/c mice), serum antibodies were only detected against the $b s g A$-derived portion of the fusion protein, not to the $\beta$-galactosidase domain.

Using the $b s g A$-specific monoclonal antibody in Western blot experiments, we demonstrated the presence of the $b s g A$ protein in both vegetatively growing and developing $M y x o-$ coccus cells. These observations are consistent with our previous finding that mutations in the $b s g A$ gene have an observable effect on the behavior of cells during both the vegetative and developmental phases of the Myxococcus life cycle. The level of $b s g A$ protein in total cell extracts remained fairly constant in vegetatively growing cells and developing cells harvested at various times up to the onset of fruiting body formation. During this time, several events in the developmental program, including the transcription of several preaggregation, development-specific genes as well as fruiting body formation itself, are dependent upon the $b s g A$ protein. The gradual twofold drop in the amount of the protein that is detected during this time as the cells enter into the developmental program may simply be due to physiological adaptations to starvation or to the increased activity of proteases in the cell extracts. Indeed, we have found that the bsgA protein is very sensitive to proteolytic degradation in cell extracts and, further, that the level of proteolytic activity increases significantly during development (data not shown). There is certainly no evidence that this protein exerts its effect on developmental gene expression after an increase in its level in the cell, as is the case for the $E$. coli $r e c A$ and $h t p R$ proteins, which modulate the SOS and heat shock responses, respectively.

We have also used the $b s g A$-specific monoclonal antibody and Western blot analysis to determine the location of the $b s g A$ protein in vegetatively growing cells. Our results suggest that at least the majority of this protein is found in the cytoplasmic fraction. It was not detected in the culture supernatant or the periplasmic fraction of osmotically shocked cells. A small proportion, 5 to $10 \%$, of the protein was found in the crude membrane fraction. However, the material that was found in the crude membrane fraction may not simply be contamination by soluble protein. Interestingly, when this crude membrane material was further fractionated by equilibrium sucrose gradient centrifugation, the $b s g A$ protein in this fraction banded at the densities previously reported for the $M$. xanthus inner membrane and, to a lesser extent, the hybrid membrane. It was not detected in either the outer membrane fraction or at the location of $\beta$-galactosidase (data not shown), taken to be representative of soluble proteins. This suggests the intriguing possibility that the membrane association may indeed represent a functional association.

\section{ACKNOWLEDGMENTS}

We are indebted to Brian Regis for assistance with isolating the hybridomas.

This work was supported by Public Health Service grant GM31900 from the National Institute of General Medical Sciences awarded to R.E.G.

\section{LITERATURE CITED}

1. Amann, E., and J. Brosius. 1985. 'ATG vectors' for regulated high-level expression of cloned genes in Escherichia coli. Gene 40:183-190.

2. Berman, M. L. 1983. Vectors for constructing hybrid genes. Biotechniques 1:178-183.

3. Casadaban, M. J., J. Chou, and S. N. Cohen. 1980. In vitro gene fusions that join an enzymatically active $\beta$-galactosidase segment to amino-terminal fragments of exogenous proteins: Escherichia coli plasmid vectors for the detection and cloning of translational initiation signals. J. Bacteriol. 143:971-980.

4. Davis, R. W., D. Botstein, and J. R. Roth. 1980. A manual for genetic engineering: advanced bacterial genetics. Cold Spring Harbor Laboratory, Cold Spring Harbor, N.Y.

5. Devi, A. L., and H. D. McCurdy. 1984. Cyclic GMP and cyclic AMP binding proteins in Myxococcus xanthus. J. Gen. Microbiol. 130:1845-1849.

6. Germino, J., J. G. Gray, H. Charbonneau, T. Vanaman, and D. Bastia. 1983. Use of gene fusions and protein-protein interaction in the isolation of a biologically active regulatory protein: the replication initiator protein of plasmid R6K. Proc. Natl. Acad. Sci. USA 80:6848-6852.

7. Gill, R., and M. Cull. 1986. Control of developmental gene expression by cell-to-cell interactions in Myxococcus xanthus. J. Bacteriol. 168:341-347.

8. Gill, R. E., M. G. Cull, and S. Fly. 1988. Genetic identification and cloning of a gene required for developmental cell interactions in Myxococcus xanthus. J. Bacteriol. 170:5279-5288.

9. Hagen, D. C., A. P. Bretscher, and D. Kaiser. 1978. Synergism between morphogenetic mutants of Myxococcus xanthus. Dev. Biol. 64:284-296.

10. Hancock, K., and V. C. W. Tsang. 1983. India ink staining of proteins on nitrocellulose paper. Anal. Biochem. 133:157-162.

11. Hodgkin, J., and D. Kaiser. 1977. Cell-to-cell stimulation of movement in non-motile mutants of Myxococcus. Proc. Natl. Acad. Sci. USA 74:2938-2942.

12. Janssen, G., and M. Dworkin. 1985. Cell-cell interactions in developmental lysis of Myxococcus xanthus. Dev. Biol. 112: 194-202.

13. Johnson, D. A., J. A. Gautsch, J. R. Sportsman, and J. H. Elder. 1984. Improved technique utilizing non-fat dried milk for analysis of proteins and nucleic acids transferred to nitrocellulose. Gene Anal. Tech. 1:3-8.

14. Kennett, R. H., T. J. McKearn, and K. B. Bechtol (ed.). 1980. Monoclonal antibodies. Plenum Publishing Corp., New York.

15. Kroos, L., and D. Kaiser. 1987. Expression of many developmentally regulated genes in Myxococcus depends on a sequence 
of cell interactions. Genes Dev, 1:840-854.

16. Kroos, L., A. Kuspa, and D. Kaiser. 1986. A global analysis of developmentally regulated genes in Myxococcus xanthus. Dev. Biol. 117:252-266.

17. Kuner, J., and D. Kaiser. 1982. Fruiting body morphogenesis in submerged cultures of Myxococcus xanthus. J. Bacteriol. 151: $485-461$.

18. Kuspa, A., L. Kroos, and D. Kaiser. 1986. Intercellular signalling is required for developmental gene expression in Myxococcus xanthus. Dev. Biol. 117:267-276.

19. Laemmli, U. K. 1970. Cleavage of structural proteins during the assembly of the head of bacteriophage T4. Nature (London) 227:680-685.

20. LaRossa, R., J. Kuner, D. Hagan, C. Manoil, and D. Kaiser. 1983. Developmental cell interactions of Myxococcus xanthus: analysis of mutants. J. Bacteriol. 153:1394-1404.

21. Maniatis, T., E. F. Fritsch, and J. Sambrook. 1982. Molecular cloning: a laboratory manual. Cold Spring Harbor Laboratory, Cold Spring Harbor, N.Y.

22. Oliver, D. B., and J. Beckwith. 1982. Regulation of a membrane component required for protein secretion in Escherichia coli. Cell 30:311-319.

23. Orndorff, P. E., and M. Dworkin. 1980. Separation and properties of the cytoplasmic and outer membranes of vegetative cells of Myxococcus xanthus. J. Bacteriol. 141:914-927.

24. Osborne, M. J., J. E. Gander, E. Parisi, and J. Carson. 1972. Mechanism of assembly of the outer membrane of Salmonella typhimurium. Isolation and characterization of cytoplasmic and outer membrane. J. Biol. Chem. 247:3962-3972.

25. Rosenberg, E. 1984. Myxobacteria development and cell interactions. Springer-Verlag, New York.

26. Sadler, J. R., M. Tecklenburg, and J. L. Betz. 1980. Plasmids containing many tandem copies of a synthetic lactose operator. Gene 8:279-300.

27. Sancar, A., A. M. Hack, and W. D. Rupp. 1979. Simple method for identification of plasmid-coded proteins. J. Bacteriol. 137: $692-693$.

28. Shimkets, L. J. 1987. Control of morphogenesis in myxobacteria. Crit. Rev. Microbiol. 14:195-227. 